\title{
Dependence of the electronic structure of $\mathrm{SrRuO}_{3}$ and its degree of correlation on cation off-stoichiometry
}

\author{
Wolter Siemons, ${ }^{1,2}$ Gertjan Koster, ${ }^{1, *}$ Arturas Vailionis, ${ }^{1}$ Hideki Yamamoto, ${ }^{1,3}$ Dave H. A. Blank, ${ }^{2}$ and Malcolm R. Beasley ${ }^{1}$ \\ ${ }^{1}$ Geballe Laboratory for Advanced Materials, Stanford University, Stanford, California 94305, USA \\ ${ }^{2}$ Faculty of Science and Technology and MESA + Institute for Nanotechnology, University of Twente, P.O. Box 217, \\ 7500 AE Enschede, The Netherlands \\ ${ }^{3}$ NTT Basic Research Laboratories, 3-1 Wakamiya Morinosato, Atsugi-shi, Kanagawa 243-0198, Japan
}

(Received 2 February 2007; revised manuscript received 21 May 2007; published 24 August 2007)

\begin{abstract}
We have grown and studied high quality $\mathrm{SrRuO}_{3}$ films grown by molecular beam epitaxy as well as pulsed laser deposition. By changing the oxygen activity during deposition, we were able to make $\mathrm{SrRuO}_{3}$ samples that were stoichiometric (low oxygen activity) or with ruthenium vacancies (high oxygen activity). This ability to control the ruthenium stoichiometry has permitted us to make a systematic study of the dependence of the degree of electron correlation in $\mathrm{SrRuO}_{3}$ on stoichiometry using transport and photoemission experiments. We have compared the measured ultraviolet photoemission spectroscopy spectra with calculated density of states spectra and offer explanations for the large observed differences between the two.
\end{abstract}

DOI: 10.1103/PhysRevB.76.075126

\section{INTRODUCTION}

$\mathrm{SrRuO}_{3}$ is a material of uncommon scientific interest. It is unusual among the correlated complex oxides in being a metallic itinerant ferromagnet. It exhibits so-called bad metal behavior at high temperatures, but is clearly a Fermi liquid at low temperatures. It is also of widespread utility as a conducting electrode in many devices utilizing complex oxides of various kinds. One of the important open questions in the physics of $\mathrm{SrRuO}_{3}$ is its degree of electron correlation and how that correlation affects the physical properties of the material. A related question is what factors control the degree of correlation. Recently Kim et al. ${ }^{1}$ have shown using photoemission data how the degree of correlation varies systematically in the ruthenate family of complex oxides as a whole. Toyota et al., ${ }^{2,3}$ using transport and photoemission measurements, have studied the degree of correlation as a function of $\mathrm{SrRuO}_{3}$ film thickness (at the few monolayer level) and shown that a correlation-driven insulator-to-metal transition occurs as film thickness increases. In this paper, we demonstrate that the degree of correlation depends on stoichiometry and show how this dependence correlates with various physical properties of the material.

At the same time, our results solve the heretofore mystery of why the transport properties of $\mathrm{SrRuO}_{3}$ are so sensitive to how it is synthesized, particularly in thin film form. ${ }^{4}$ More specifically, we show that this sensitivity is not a simple matter of defect scattering but, rather, once again is related to stoichiometry and the degree of correlation.

The properties observed in the ruthenate family of complex oxides range from very good metals $\left(\mathrm{RuO}_{2}\right)$ to insulators $\left(\mathrm{Y}_{2} \mathrm{Ru}_{2} \mathrm{O}_{7}\right)$. Recently, Kim et al. ${ }^{1}$ suggested that this change in electronic behavior can be attributed to a change in electron-electron correlation. They come to this conclusion based on fits of experimental core-level photoemission spectra [x-ray photoemission spectroscopy (XPS), Cox et al. ${ }^{5}$ ] using dynamic mean field theory. ${ }^{6}$ The $3 d$ peaks of ruthenium are compared for ruthenates put in order of metallicity, and a systematic relative shift in spectral weight from the so-called screened peak to the unscreened peak is observed.
PACS number(s): 73.50.-h

To explain these shifts, these authors ${ }^{1}$ use a model in which the fitting parameters are the Hubbard $U$ and the bandwidth $W$ of the ruthenium $4 d$ band. The outcome of their analysis is that the ratio $U / W$ correlates with the ratio of the screened and unscreened peaks, from which one can deduce that the stronger the screened peak, the less correlation (i.e., the more metallic).

In ultraviolet photoemission spectroscopy (UPS) spectra, a similar shift in spectral weight is observed. In this case, the $t_{2 g}$ peak, which is very close to the Fermi level and referred to as the coherent peak (i.e., the quasiparticle band near $E_{F}$ ), is reduced as correlation increases, and a broad peak around $1.5 \mathrm{eV}$ starts to rise. This latter peak is called the incoherent peak (i.e., the remnant of the Hubbard bands $1-2 \mathrm{eV}$ above and below $E_{F}$ ). Both $\mathrm{SrRuO}_{3}$ and $\mathrm{CaRuO}_{3}$ can be classified by their XPS and UPS spectra in this way, despite, for example, similar transport properties at room temperature. Based on the classification by XPS spectra, $\mathrm{CaRuO}_{3}$ would be a more correlated system than $\mathrm{SrRuO}_{3}$. However, the samples studied in Ref. 1 could have suffered from the same off-stoichiometry as will be discussed in this paper (for instance, due to the surface or sample preparation processes).

Viewed from the perspective of the work by Kim et al., ${ }^{1}$ in this paper, we show that similar systematic variations in the degree of correlation can occur within a single ruthenate as a function of stoichiometry. In particular, it turns out that one source of disorder or off-stoichiometry can be varied in $\mathrm{SrRuO}_{3}$ thin films by changing the deposition conditions or, what turns out to be more or less equivalent, the deposition technique. Specifically, we demonstrate that variation of vacancies on the ruthenium site gives rise to a systematic change in the degree of correlation. Moreover, the transport properties of our samples are clearly linked to their photoemission spectra (XPS and UPS) and to the crystal unit cell parameters. This correlation with the unit cell volume $V_{c}$ permits us to use $V_{c}$ as a surrogate variable for the ruthenium deficiency, a dependency which is well known from literature. ${ }^{4}$ This change in volume also alters the average $\mathrm{Ru}-\mathrm{O}-\mathrm{Ru}$ bond angle, which is known to be closely connected to the $d$-band width ${ }^{7}$ and, therefore, electron correla- 
tion. $\mathrm{SrRuO}_{3}$ appears to be a system where these effects of correlation, which are normally not easily accessible, can be studied in a systematic fashion, but we suspect that the underlying physics is generic.

\section{EXPERIMENT}

The thin film samples reported in this paper are grown by two different methods: molecular beam epitaxy (MBE) and pulsed laser deposition (PLD). The samples are grown in the same vacuum chamber with a background pressure of $10^{-9}$ Torr. All films are grown on $\mathrm{TiO}_{2}$ terminated $\mathrm{SrTiO}_{3}$ substrates, prepared according to Koster et al. ${ }^{8}$ The MBE samples are deposited in an oxygen pressure of $10^{-5}$ Torr and at a substrate temperature of $700{ }^{\circ} \mathrm{C}$ at a rate of about $1 \AA / \mathrm{s}$ from separate ruthenium and strontium sources, which enables us to vary the ratio of ruthenium to strontium. Electron impact emission spectroscopy is used to control the deposition rate of the electron beam heated sources. Typical thickness of the films grown ranges from 200 to $300 \AA$. During growth, atomic oxygen is provided by an Astex SXRHA microwave plasma source. By adjusting oxygen flow and generator wattage $(200-600 \mathrm{~W})$, the amount of atomic oxygen can be controlled. ${ }^{9}$ During growth, reflection highenergy electron diffraction (RHEED) is used to monitor the morphology of the samples.

For PLD a $\mathrm{KrF}$ excimer laser produces a $248 \mathrm{~nm}$ wavelength beam with typical pulse lengths of 20-30 ns. A rectangular mask shapes the beam and a variable attenuator permits variation of the pulse energy. The energy density on the target is kept at approximately $2.1 \mathrm{~J} / \mathrm{cm}^{2}$. Before each run the rotating stoichiometric $\mathrm{SrRuO}_{3}$ target is preablated for $2 \mathrm{~min}$ at $4 \mathrm{~Hz}$. Films are deposited with a laser repetition rate of $4 \mathrm{~Hz}$, with the substrate temperature at $690^{\circ} \mathrm{C}$. The oxygen background pressure was $10^{-5}$ Torr as in the MBE case; however, no atomic oxygen was provided for the PLD films. The thickness of the films is in the same range as for the MBE samples.

$\mathrm{X}$-ray diffraction (XRD) experiments were performed on a Panalytical X'pert thin film diffractometer to determine both the thickness and the crystalline quality of the samples. UPS measurements were performed in situ in a vacuum chamber attached to the main growth chamber, which has a base pressure of $<5 \times 10^{-10}$ Torr, with a VG Scientific ESCAlab Mark II system (nonmonochromatic, helium discharge). XPS measurements were performed in situ with the same system (nonmonochromatic $\mathrm{Al} K \alpha$ ). This experimental setup is crucial for obtaining reliable data since the surface of $\mathrm{SrRuO}_{3}$ is known to change when exposed to air ${ }^{10}$ in a way that gives rise to surface states in the spectra. Electrical transport properties were measured with a Quantum Design Physical Property Measurement System.

By varying the deposition conditions, we found that the properties of the films changed. The films so obtained were divided into three groups: ruthenium rich, near stoichiometric, and ruthenium poor. The ruthenium rich samples are typically made by using a relatively low oxygen partial pressure and/or low atomic oxygen flux and depositing a little excess ruthenium. The nearly stoichiometric samples are

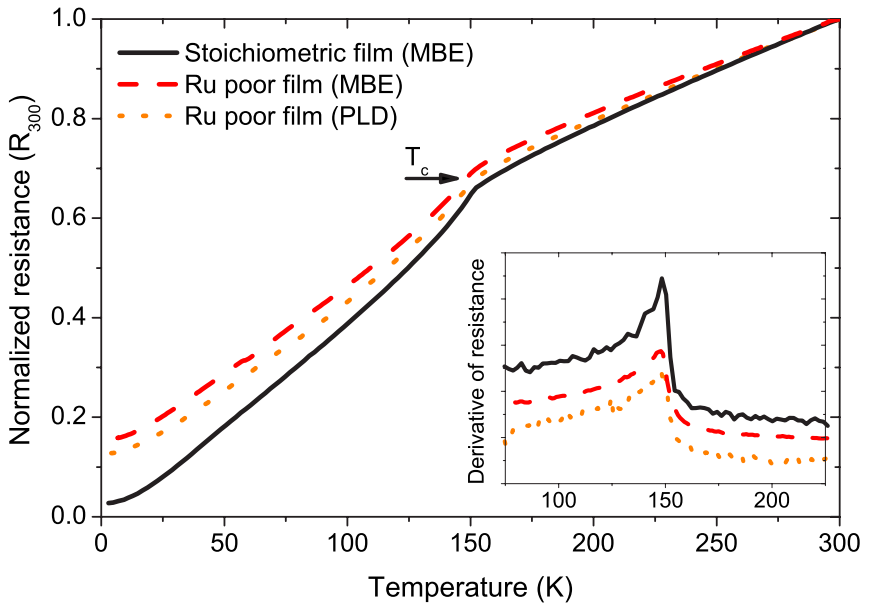

FIG. 1. (Color online) Resistance behavior as a function of temperature for a nearly stoichiometric MBE grown (black solid), a ruthenium poor MBE grown (red dashed), and a PLD grown (orange dotted) $\mathrm{SrRuO}_{3}$ film. The resistance has been normalized with respect to the values at $300 \mathrm{~K}$. The inset shows the derivative of each line; $T_{c}$ has been set as the point where the derivative is maximal. The resistivity $(\rho)$ of the stoichiometric $\mathrm{MBE}$ grown $\mathrm{SrRuO}_{3}$ at room temperature is about $190 \mu \Omega \mathrm{cm}$ and its carrier density at $4 \mathrm{~K}$ is $2 \times 10^{22} \mathrm{~cm}^{-3}$.

single phase and are obtained by tuning the oxygen activity together with the use of a $\mathrm{Ru} / \mathrm{Sr}$ ratio close to 1 . The ruthenium poor samples are created by using relatively high partial oxygen pressures and a $\mathrm{Ru} / \mathrm{Sr}$ ratio greater than or equal to 1 (the ruthenium flux does not seem to be the determining factor here).

It turns out to be impossible to grow $\mathrm{SrRuO}_{3}$ with strontium vacancies. It is possible though to grow samples that are stoichiometric or with ruthenium vacancies (for reasons that will be discussed later). These samples are single phase and have the $\mathrm{SrRuO}_{3}$ crystal structure with no precipitations. The precise stoichiometry is difficult to determine, but, based on the combined analysis (XRD and XPS), we can make the above distinction without further specifying the exact composition of individual samples in each group. All three sample types can be obtained by MBE, but samples made by PLD using a stoichiometric target are typically ruthenium poor. In the remainder of this paper, we will focus on samples that are either (near) stoichiometric or ruthenium poor.

\section{RESULTS}

We first present our resistivity measurements. In Fig. 1, the normalized resistance is plotted as a function of temperature for three samples: a stoichiometric MBE, a ruthenium poor MBE, and a ruthenium poor PLD film. The normalization factor is the room temperature resistivity. For the stoichiometric samples the room temperature resistivity (in the bad metal regime) is around $190 \mu \Omega \mathrm{cm}$, which compares well to values found in literature for polycrystalline and single crystal samples $(150-200 \mu \Omega \mathrm{cm})$. When ruthenium vacancies are introduced, the value increases markedly to roughly $300 \mu \Omega \mathrm{cm}$. The room temperature resistivity varies 

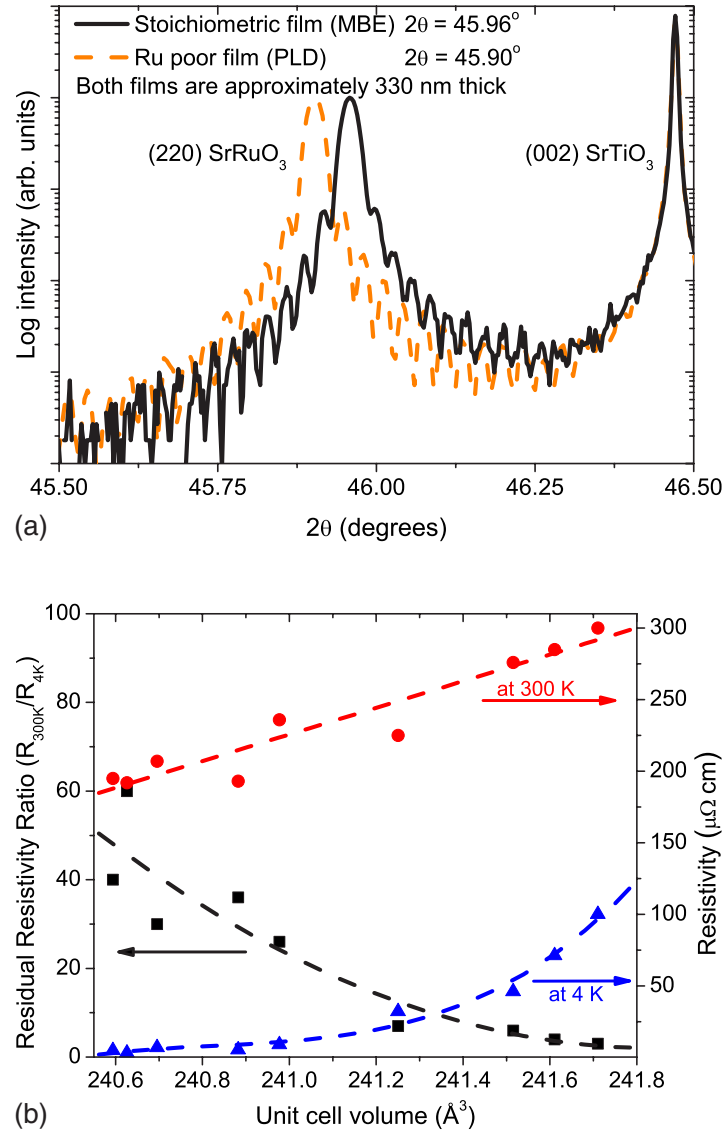

FIG. 2. (Color online) (a) Comparison of $\theta / 2 \theta$ XRD scans of two thick $(330 \mathrm{~nm}$ ) films grown by PLD (orange dashed) and MBE (black solid). The fringes indicate good crystalline quality of the films; the shift in peak position indicates that the PLD film has a larger out-of-plane lattice parameter [(110 direction)]. (b) A comparison between the volume of the $\mathrm{SrRuO}_{3}$ unit cell in thin films on $\mathrm{SrTiO}_{3}$ substrates grown by either MBE or PLD and their transport properties: room temperature (red circles) and low temperature resistivity (blue triangles) on the right $y$ axis and residual resistance ratio (black squares) on the left $y$ axis. The dashed lines are guides for the eyes.

systematically with the amount of ruthenium vacancies, as shown in Fig. 2(b) (discussed below). Also of interest is the residual resistivity ratio (RRR), which is defined as the resistivity at $300 \mathrm{~K}$ divided by the value at $4 \mathrm{~K}$. For the best single crystals, values between 50 and 100 are reported, ${ }^{11}$ and similar results can be obtained for optimized thin films. ${ }^{12}$ Like the room temperature resistivity, the RRR is very sensitive to changes in stoichiometry. For the stoichiometric sample in Fig. 1 (black solid line), the RRR is 26, which is excellent for the thickness of these samples, whereas for both ruthenium poor films (orange dotted and red dashed lines), the RRR drops to around 7. Note that for the majority of PLD films in the literature, which fall in the ruthenium poor class (see below), values of 5 or less are reported. ${ }^{13,14}$

Next we turn to the Curie temperature of our films, which are shown in the inset of Fig. 1, where the temperature derivatives of the resistance near the transition temperature are compared. The transition temperature $T_{C}$ in bulk samples is $160 \mathrm{~K}$, whereas in all of our thin film samples, $T_{C}$ is reduced by about $10 \mathrm{~K}$ (Ref. 15) due to strain. From literature, we know that when a sufficient amount of ruthenium vacancies are introduced, $T_{C}$ is lowered further. ${ }^{4}$ This gives us some indication of the order of magnitude of the vacancy density. On this basis, we estimate that for the range of samples we studied, the vacancy concentration is much smaller than a few percent.

In order to get a quantitative indicator of the ruthenium deficiency, we examined the change in lattice parameters in going from the stoichiometric to ruthenium poor samples. In Fig. 2(a), we show $\theta / 2 \theta$ scans for two thick films $(330 \mathrm{~nm})$, one grown with $\mathrm{MBE}$ in low oxygen pressure and one with PLD in high oxygen pressure, to illustrate the difference in the $d$ spacing for the out-of-plane (110) direction of $\mathrm{SrRuO}_{3}$ when different deposition techniques are used. These films are much thicker than the samples compared in other parts of the paper to show that the films remain perfectly crystalline and strained up to very large thicknesses. In addition, these scans reveal the very high degree of crystallinity of the samples by the existence of finite size fringes in the scans for both samples. As can be seen, the out-of-plane lattice constant is larger for the film grown in high oxygen pressures, explained by a lower ruthenium content. ${ }^{4}$ Since the $c$ axis (in plane) of the $\mathrm{SrRuO}_{3}$ is fixed by epitaxy (the films are fully strained for all sample thicknesses in this paper), the increase in the (110) direction (linear combination of the $a$ and $b$ axes) is directly proportional to an increase in unit cell volume, which we take as our measure of the ruthenium deficiency in Fig. 2(b).

We obtained the lattice parameters by refining on six reflections. For samples which were classified as having low ruthenium content, we find a unit cell with larger volume. This increase in the case of ruthenium vacancies is consistent with earlier measurements done on ruthenium deficient single crystal samples. ${ }^{4}$ The bulk values taken from literature are $a=5.572 \AA, \quad b=5.533 \AA, \quad c=7.849 \AA$, and $\mathrm{V}_{b u l k}$ $=242.0 \AA^{3}$ (Ref. 4) for single crystals and correspond to an unstrained lattice, unlike the films grown here on $\mathrm{SrTiO}_{3}$.

In Fig. 2(b), we show how changes in the transport data correlate with the volumes of the orthorhombic unit cell for various films studied. The room temperature and low temperature resistivities as well as the RRRs show a clear trend, with smaller volumes showing the lowest resistivities and highest RRRs. These films are typically found in our second group made by MBE (oxygen activity tuned to optimize oxidation and ruthenium sticking). On the other end, PLD films appear to have larger volumes and poorer transport properties. Note that the Curie temperature should also show a similar trend, although the change in $T_{C}$ is small as shown in Fig. 1.

To get a clearer understanding of how or whether the electronic properties of $\mathrm{SrRuO}_{3}$ vary as a function of stoichiometry, we measured the UPS and XPS spectra of some of our samples. In Fig. 3(a), the UPS spectra are plotted for the same samples that were used for the transport data in Fig. 1. First, for the stoichiometric $\mathrm{SrRuO}_{3}$ sample grown by MBE, the spectrum shows a peak at the Fermi energy, corresponding to the ruthenium $t_{2 g}$ band and a valley at binding energy $\sim 1.5 \mathrm{eV}$. This peak has been observed before by Kim et al. ${ }^{16}$ and is also expected based on models. ${ }^{17}$ In ruthenium poor 

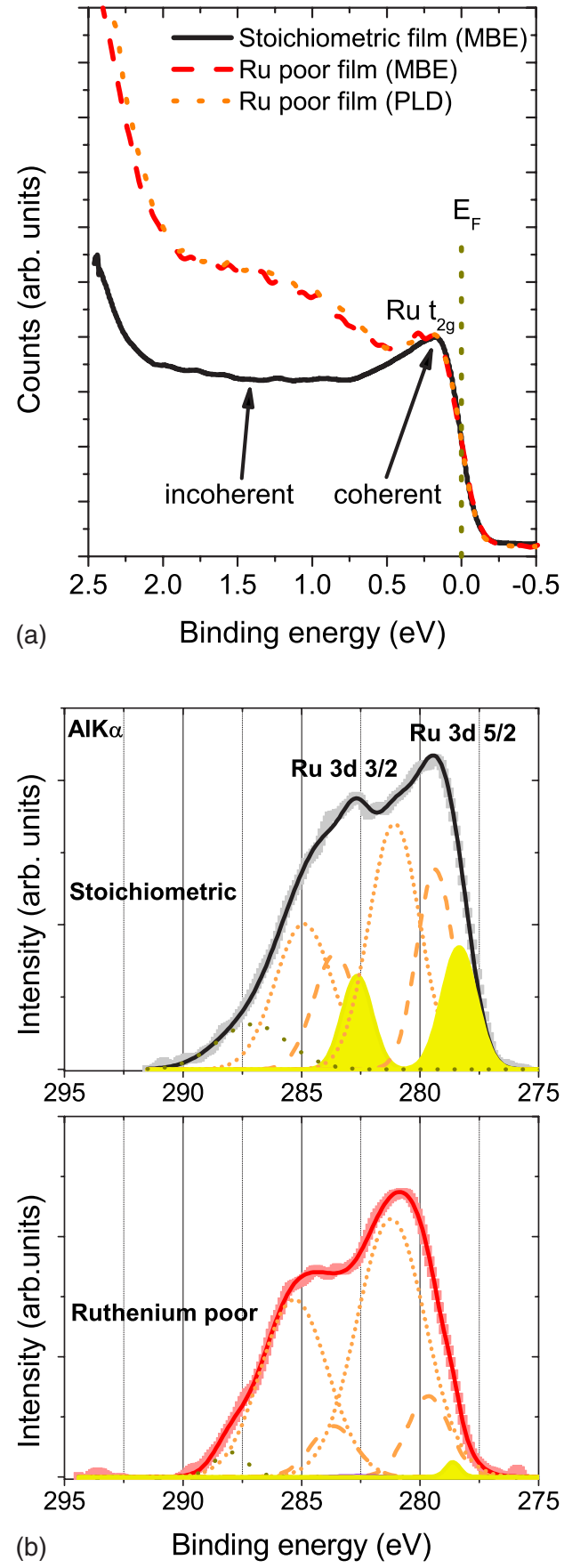

FIG. 3. (Color online) (a) UPS spectra at room temperature of three $\mathrm{SrRuO}_{3}$ films: a MBE film (black solid), a ruthenium poor MBE film (red dashed), and a PLD film (orange dotted) (same samples as in Fig. 1). All samples show states at the Fermi edge and a Ru $t_{2 g}$ peak close to the Fermi level. The spectra have been normalized on the $t_{2 g}$ peak. (b) XPS detail spectra at room temperature of the Ru $3 d$ doublet of a stoichiometric film (top) and a ruthenium poor film (bottom) including fitting of the spectra using three spin orbit coupled doublets represented by Gaussians (two pairs orange dashed or dotted, the third pair yellow filled); the overlapping $\operatorname{Sr} 2 p_{1 / 2}$ peak (Gaussian) was subtracted for both spectra. In the spectrum of stoichiometric film, more pronounced screened peaks at lower binding energy (yellow filled) are needed to give a good fit, indicative of less correlated behavior. For the ruthenium poor spectrum, the screened peaks are almost nonexistent. samples, as can be seen in Fig. 3(a), for both the PLD film and the ruthenium poor MBE film, a second, broad peak appears at $\sim 1.5 \mathrm{eV}$, the so-called incoherent peak. Note that these spectra are normalized on the $t_{2 g}$ peak to make their comparison easier.

Examples of XPS core-level spectra for one stoichiometric sample and one ruthenium poor sample are plotted in Fig. 3(b). For clarity, we reduced these spectra of the ruthenium $3 d$ doublet by subtracting the strontium $3 p_{1 / 2}$ peak fitted with a Gaussian, which overlaps at lower binding energy. Note that our in situ measurements are free of carbon contamination and its complications near the surface region. ${ }^{10}$ This is confirmed by the absence of the $\mathrm{C} 1 \mathrm{~s}$ peak, which normally also overlaps with the $\mathrm{Ru} 3 d$ peaks. The stoichiometric sample shows more spectral weight for the $\mathrm{Ru} 3 d$ peaks at lower binding energy compared to the ruthenium poor sample. Peak fitting using three sets of Gaussians (the area of the peaks in each spin orbit doublet pair have a 2:3 ratio) shows that at a binding energy of $\sim 278 \mathrm{eV}$ an extra curve (yellow filled) is necessary to fit the data for stoichiometric $\mathrm{SrRuO}_{3}$, which is arguably the so-called screened peak typical for $\mathrm{SrRuO}_{3}$. On the other hand, the data for the ruthenium poor sample are dominated by the unscreened peak.

\section{DISCUSSION}

Before discussing the data explicitly, we would first like to point out that the stoichiometry of the samples is extremely dependent on the oxygen activity during deposition. For MBE, this can be independently varied by controlling the flux of molecular or atomic oxygen. Apparently, the sticking of ruthenium (possibly through the formation of $\mathrm{RuO}_{4}$, which is very volatile ${ }^{18,19}$ ) is a function of oxygen activity: at relatively low oxygen activity, the stoichiometry is mostly determined by the supplied $\mathrm{Sr} / \mathrm{Ru}$ ratio. When excess ruthenium is supplied, RHEED, scanning electron microscopy, Auger spectroscopy, and transmission electron microscopy reveal precipitation of $\mathrm{RuO}_{2}$, and the $\mathrm{Ru} 3 d$ corelevel spectra show very strong screened peaks typical for $\mathrm{RuO}_{2}$. At intermediate oxygen activity it is most favorable to achieve perfect stoichiometry: RHEED shows two dimensional growth and XPS and/or UPS show almost perfect spectra typical for $\mathrm{SrRuO}_{3}$. The "best" values for resistivity and RRR are obtained here. Finally, at high oxygen activity ruthenium vacancies are unavoidable, independent of the ratio $\mathrm{Sr} / \mathrm{Ru}$ in the supplied vapor: RHEED shows two dimensional growth, layer by layer or even step flow, ${ }^{20}$ and XRD clearly shows a modification in the unit cell, indicative of ruthenium vacancies. $^{4}$

We believe the last scenario to be the case also for PLD films, where a high (atomic) oxygen pressure exists within the plume (in addition to the background activity) and very little can be done to avoid this. PLD offers the advantage of making the same quality film every time, albeit ruthenium poor. On the other hand, we have shown that, even when ruthenium vacancies are present, the crystallinity of the material remains the same compared to the stoichiometric films and therefore cannot be used to explain any of the observed variations in transport and photoelectron spectroscopy (PES). 
There seems no indication that oxygen vacancies play a significant role, but we cannot distinguish this from the formation of ruthenium vacancies by looking at the response of lattice constants only. In a study of similar ruthenium deficient samples, oxygen vacancies were not observed. ${ }^{4}$

Resistivity is affected both at low temperatures with lower RRR values and at higher temperatures with a variation in lower room temperature resistivity for stoichiometric samples. At low temperatures, defect scattering dominates transport properties, resulting in the systematic behavior of the resistivity at low temperature and the RRR values, increasing and decreasing, respectively, almost exponentially with unit cell volume (more defects). On the other hand, at room temperature in the bad metal regime, the resistivity increases more linearly with the unit cell volume. This indicates that more than just scattering is contributing at room temperature. It would be interesting to study the high temperature behavior (beyond the Ioffe-Regel limit ${ }^{21}$ ) as a function of stoichiometry, which is part of a planned future endeavor.

Now we turn to the observed differences in PES and their correlation with transport properties as a function of ruthenium stoichiometry. First, it is logical to exclude the ruthenium rich samples from this comparison because both transport and PES would be a mixture of $\mathrm{SrRuO}_{3}$ and precipitated $\mathrm{RuO}_{2}$. Both UPS and XPS spectra of films with ruthenium vacancies are distinctly different from their stoichiometric counterparts. Our data of the $\mathrm{Ru} 3 d$ peaks in $\mathrm{SrRuO}_{3}$ with vacancies suggest that these ruthenium poor samples show almost no screened peaks, whereas the stoichiometric samples show pronounced screened peaks. This effect is most often associated with the material becoming more correlated, which has been quantified by Kim et al. $;^{1}$ see also our earlier discussion. In the same spirit, ruthenium poor samples show spectral weight at $\sim 1.5 \mathrm{eV}$ in He I UPS, which has previously been attributed to an incoherent peak in a picture of a strongly correlated system. When the $\sim 1.5 \mathrm{eV}$ peak is absent, the $t_{2 g}$ peak at the Fermi level becomes more pronounced. It is noteworthy here that the $t_{2 g}$ peak at the Fermi level has not yet been observed with scraped bulk samples, indicating that thin film specimens may provide a better opportunity in investigating the intrinsic electronic structure of $\mathrm{SrRuO}_{3}$ with photoemission spectroscopy as has been pointed out by Kim et al. ${ }^{16}$ This shift in spectral weight is to be expected when the spectra are compared with density of states (DOS) calculations. ${ }^{17,22,23}$ The calculations show a $t_{2 g}$ peak with almost the same height as the $\mathrm{O} 2 p$ peak. We can consider this discrepancy in terms of a self-energy correction to the one-electron band structure. ${ }^{7,16,17,23,24}$ Due to the $k$ dependence of the self-energy, the spectral weight at the Fermi level is reduced by a factor $m_{k} / m_{b}$, where $m_{k}$ is called the " $k$ mass" and $m_{b}$ is the bare band mass. By comparing with band calculations, we estimate $m_{k} / m_{b}$ to be 0.3 for the sample with ruthenium vacancies and 0.6 for the stoichiometric sample. Combining these values with the mass enhancement factor $\mathrm{m}^{*} / m_{b}$ as derived from the electronic specific heat $\gamma$, one obtains the quasiparticle weight $Z=\left(m_{k} / m_{b}\right) /\left(m^{*} / m_{b}\right)$. For $m^{*} / m_{b}$, we have used the average value of 4.1 as determined by two different measurements. ${ }^{17,22}$ This leads to values of $Z^{-1}$ of 13 and 7 for the ruthenium poor and stoichiometric samples, respectively. The value of 13 for the ruthenium poor samples is in line with what has been reported by most authors. $16,17,23$ The value of 7 for the stoichiometric samples is slightly lower than what was reported by Kim et al. ${ }^{16}$ on their samples. The high values for $Z^{-1}$ have been used by others ${ }^{17,23}$ to explain the reduction of the $t_{2 g}$ peak at the Fermi level due to electron correlation. Our work shows that the intensity of the $t_{2 g}$ peak is very sensitive to the ruthenium stoichiometry and that UPS spectra of stoichiometric films look much more like the calculated DOS than ruthenium poor films. We conclude that ruthenium stoichiometry (and possibly surface states contribute as well) has a much larger impact on PES spectra than previously assumed and could provide for a large part an explanation for the observed photoemission results in this paper, but also by others. Of course, the change in the PES spectra could be caused by a change in correlation due to the change in unit cell and the Ru-O-Ru bond angle, but it is also possible that the vacancies themselves caused it. Therefore we propose that besides a change in correlation, the transfer of spectral weight could also be caused by inelastic processes at the ruthenium vacancies. The $t_{2 g}$ orbitals that form the conduction band form states spreading in two dimensions. To define the energy of a state to within the observed $0.5 \mathrm{eV}$ peak, it would be necessary to construct a packet of band states, which, for a band of width $4 \mathrm{eV}$, would extend $4 / 0.5=8$ cube edges. The corresponding $\pi 4^{2} \approx 50$ ruthenium sites would generally contain at least one vacancy, even at only $2 \%$ vacancies. It would be reasonable to expect that the removal of a band electron might cause shake-off excitations, vibrational or electronic, of the vacancy. This would increase the energy needed to eject the electron, as can be seen in both the UPS and XPS spectra.

\section{CONCLUSIONS}

We have grown $\mathrm{SrRuO}_{3}$ thin films on $\mathrm{SrTiO}_{3}$ under various conditions and conclude that this material exhibits a range of properties due to a subtle change in stoichiometry on the ruthenium site related to the oxidation conditions during deposition. Resistivity, x-ray diffraction, UPS, and XPS all seem to indicate that this change in behavior is due to a changing electron-electron correlation, although we also point out that contributions from inelastic processes at vacancy sites as well as from surface states cannot be ignored. These results shed light on the well-known sensitivity of the properties of $\mathrm{SrRuO}_{3}$ to its synthesis conditions. Equally important, they suggest a clear path to more quantitative comparisons with theory.

\section{ACKNOWLEDGMENTS}

One of us (G.K.) thanks the Netherlands Organization for Scientific Research (NWO, VENI). W.S. thanks the Nanotechnology network in the Netherlands, NanoNed. We would also like to thank Walt Harrison, M. Naito, Kookrin Char, Ann Marshall, Jim Reiner, Guus Rijnders, Ted Geballe, and Robert Hammond for helpful discussions. This work was supported by the DOE BES with additional support from EPRI. 
*Corresponding author. g.koster@utwente.nl

${ }^{1}$ H. D. Kim, H. J. Noh, K. H. Kim, and S. J. Oh, Phys. Rev. Lett. 93, 126404 (2004).

${ }^{2}$ D. Toyota, I. Ohkubo, H. Kumigashira, M. Oshima, T. Ohnishi, M. Lippmaa, M. Kawasaki, and H. Koinuma, J. Appl. Phys. 99, 08N506 (2006).

${ }^{3}$ D. Toyota, I. Ohkubo, H. Kumigashira, M. Oshima, T. Ohnishi, M. Lippmaa, M. Takizawa, A. Fujimori, K. Ono, M. Kawasaki, and H. Koinuma, Appl. Phys. Lett. 87, 162508 (2005).

${ }^{4}$ B. Dabrowski, O. Chmaissem, P. W. Klamut, S. Kolesnik, M. Maxwell, J. Mais, Y. Ito, B. D. Armstrong, J. D. Jorgensen, and S. Short, Phys. Rev. B 70, 014423 (2004).

${ }^{5}$ P. A. Cox, R. G. Egdell, J. B. Goodenough, A. Hamnett, and C. C. Naish, J. Phys. C 16, 6221 (1983).

${ }^{6}$ A. Georges, G. Kotliar, W. Krauth, and M. J. Rozenberg, Rev. Mod. Phys. 68, 13 (1996).

${ }^{7}$ M. Imada, A. Fujimori, and Y. Tokura, Rev. Mod. Phys. 70, 1039 (1998).

${ }^{8}$ G. Koster, B. L. Kropman, G. J. H. M. Rijnders, D. H. A. Blank, and H. Rogalla, Appl. Phys. Lett. 73, 2920 (1998).

${ }^{9}$ N. J. C. Ingle, R. H. Hammond, M. R. Beasley, and D. H. A. Blank, Appl. Phys. Lett. 75, 4162 (1999).

${ }^{10}$ J. Shin, S. V. Kalinin, H. N. Lee, H. M. Christen, R. G. Moore, E. W. Plummer, and A. P. Baddorf, Surf. Sci. 581, 118 (2005).

${ }^{11}$ G. Cao, S. McCall, M. Shepard, J. E. Crow, and R. P. Guertin, Phys. Rev. B 56, 321 (1997).

${ }^{12}$ L. Klein, J. S. Dodge, C. H. Ahn, J. W. Reiner, L. Mieville, T. H. Geballe, M. R. Beasley, and A. Kapitulnik, J. Phys.: Condens.
Matter 8, 10111 (1996).

${ }^{13}$ J. P. Maria, S. Trolier-McKinstry, D. G. Schlom, M. E. Hawley, and G. W. Brown, J. Appl. Phys. 83, 4373 (1998).

${ }^{14}$ X. D. Wu, S. R. Foltyn, R. C. Dye, Y. Coulter, and R. E. Muenchausen, Appl. Phys. Lett. 62, 2434 (1993).

${ }^{15}$ Q. Gan, R. A. Rao, C. B. Eom, J. L. Garrett, and M. Lee, Appl. Phys. Lett. 72, 978 (1998).

${ }^{16}$ J. Kim, J. Chung, and S. J. Oh, Phys. Rev. B 71, 121406(R) (2005); J. Kim, J.-Y. Kim, B.-G. Park, and S.-J. Oh, Phys. Rev. B 73, 235109 (2006).

${ }^{17}$ J. Okamoto, T. Mizokawa, A. Fujimori, I. Hase, M. Nohara, H. Takagi, Y. Takeda, and M. Takano, Phys. Rev. B 60, 2281 (1999).

${ }^{18}$ M. Nakahara, S. Tsunekawa, K. Watanabe, T. Arai, T. Yunogami, and K. Kuroki, J. Vac. Sci. Technol. B 19, 2133 (2001).

${ }^{19}$ W. E. Bell and M. Tagami, J. Phys. Chem. 67, 2432 (1963).

${ }^{20}$ G. Rijnders, D. H. A. Blank, J. Choi, and C. B. Eom, Appl. Phys. Lett. 84, 505 (2004).

${ }^{21}$ A. F. Ioffe and A. R. Regel, Prog. Semicond. 4, 237 (1960).

${ }^{22}$ P. B. Allen, H. Berger, O. Chauvet, L. Forro, T. Jarlborg, A. Junod, B. Revaz, and G. Santi, Phys. Rev. B 53, 4393 (1996).

${ }^{23}$ K. Fujioka, J. Okamoto, T. Mizokawa, A. Fujimori, I. Hase, M. Abbate, H. J. Lin, C. T. Chen, Y. Takeda, and M. Takano, Phys. Rev. B 56, 6380 (1997).

${ }^{24}$ I. H. Inoue, Y. Aiura, Y. Nishihara, Y. Haruyama, S. Nishizaki, Y. Maeno, T. Fujita, J. G. Bednorz, and F. Lichtenberg, Physica B 224, 516 (1996). 